\title{
L'Europe des revues II (1860-1930). Réseaux et circulations des modèles, dir. E. STEAD et H. VÉDRINE
}

Ida Merello

\section{OpenEdition}

\section{Journals}

\section{Edizione digitale}

URL: https://journals.openedition.org/studifrancesi/23224

DOI: 10.4000/studifrancesi.23224

ISSN: 2421-5856

\section{Editore}

Rosenberg \& Sellier

\section{Edizione cartacea}

Data di pubblicazione: 1 avril 2020

Paginazione: 208-209

ISSN: 0039-2944

\section{Notizia bibliografica digitale}

Ida Merello, «L'Europe des revues I/ (1860-1930). Réseaux et circulations des modèles, dir. E. sTEAD et H. VÉDRINE», Studi Francesi [Online], 190 (LXIV | I) | 2020, online dal 01 mai 2020, consultato il 03 août 2021. URL: http://journals.openedition.org/studifrancesi/23224 ; DOI: https://doi.org/10.4000/ studifrancesi.23224

Questo documento è stato generato automaticamente il 3 août 2021.

\section{(†)

Studi Francesi è distribuita con Licenza Creative Commons Attribuzione - Non commerciale - Non opere derivate 4.0 Internazionale. 


\title{
L'Europe des revues II (1860-1930). Réseaux et circulations des modèles, dir. E. STEAD et H. VÉDRINE
}

\author{
Ida Merello
}

\section{NOTIZIA}

L'Europe des revues II (1860-1930). Réseaux et circulations des modèles, dir. E. STEAD et H. VÉDRINE, Presses Sorbonne Université, Paris, 2018, 985 pp.

1 Il volume, enciclopedico, comprende quarantatré articoli ed è dotato di una vastissima bibliografia generale, una presentazione degli autori e un indice dei nomi che da soli potrebbero rendere il volume imprescindibile. La miscellanea è divisa in sei sezioni, ciascuna delle quali preceduta da un'introduzione. Nell'impossibilità di rendere conto qui dell'insieme degli articoli, si prendono in considerazione le sei sezioni.

2 Evanghelia STEAD \& Hélène VÉDRINE (Périodiques en réseau, pp. 7-15) ricordano l'attenzione sempre crescente accordata alle riviste negli ultimi anni, non solo per la maggiore accessibilità del corpus, ma anche per una sempre maggiore consapevolezza del suo peso critico/estetico. Le riviste si distinguono anche per l'elaborazione collettiva di teorie e manifesti. Del volume precedente ( $\mathrm{La}$ Belle époque des revues, 1880-1914, 2002, ad opera di Olivier Corpet) sono ripresi gli assunti metodologici di base, ossia l'attenzione qualitativa a punti di vista effimeri ma non per questo meno significativi. Diventa fondamentale ogni volta analizzare rapporti col contesto, mentre le storie letterarie si soffermavano soltanto sui grandi autori, per coglierne le varianti, o ritrovare prestabilite categorie estetiche, trascurando la testata. L'approccio globale alle Petites revues aveva ad esempio permesso a Décaudin di individuare le linee di forza di un campo fluttuante, per comprendere il senso di un'epoca. Ma si è sviluppato anche un modello sociologico, sia in Francia (Yves Chevrefils Desbiolles), che in Spagna (sotto la direzione di Danièle Bussy Genevois) e in Gran Bretagna (quattro volumi diretti da 
Peter Brooker e Andrew Thacker, tra il 2009 e il 2013). Soprattutto la monumentale opera inglese sottrae le riviste al carattere di marginalità, per vederle invece come indizi di molteplici innovazioni, e analizzandole come sistema di scambio tra cultura minoritaria e di massa.

Nei seminari del TIGRE, dal 2004, E. STEAD si era soffermata sulla natura ibrida delle riviste, con un'analisi sia dell'impaginazione che del contenuto, utilizzando l'immagine come angolo privilegiato di informazione.

4 La scelta del periodo da analizzare (1880-1920) del TIGRE, che non seguiva l'interruzione abituale del 1914, era utile per indicare la continuità con i primi movimenti di avanguardia. Brooker e Thacker sono addirittura arrivati al 1955 per il Regno Unito e l'Irlanda, al 1960 per l'America del Nord, e al 1940 per l'Europa.

5 Rispetto all'opera inglese, STEAD e VÉDRINE valutano maggiormente il carattere polimorfo e la materialità delle riviste, con una interazione dei metodi di analisi, e il valore dell'atto culturale in relazione al libro, la stampa, gli spettacoli, indagando la rete di rapporti nazionali e internazionali. Il lavoro mantiene il carattere interdisciplinare della prima pubblicazione del Tigre, ponendo l'accento sulle riviste come epicentro dell'attività culturale. Il termine di rete, cui si fa costante riferimento, introduce in maniera naturale anche l'approccio sociologico: STEAD e VÉDRINE ne testano la fecondità euristica. Il termine non viene considerato soltanto nel suo aspetto di relazioni umane, ma anche tra le idee e le forme, e tra forze commerciali e teorie estetiche e letterarie. A contare è soprattutto il valore di scambio.

Per valutare pienamente la funzione della rete le curatrici verificano il modo in cui le petites revues interagiscono e si riuniscono, facendo circolare i modelli.

7 La prima sezione prende in considerazione la nascita e l'organizzazione formale di periodici che segnarono la storia della stampa, da un punto di vista del formato editoriale. Si risale alla nascita nel Seicento in Francia della rivista letteraria come luogo di recensione, per verificare il suo progressivo tentativo di rigore scientifico, con l'aumento di collaboratori esperti e un'uscita trimestrale per aver il tempo di valutare le opere. Tre articoli della sezione prendono in considerazione la diffusione della rivista illustrata inglese, che, nelle sue declinazioni europee, svolge un ruolo anche politico nella strutturazione dell'immaginario. L'amalgama tra le riviste si verifica in un clima di espansione della cultura visiva, e ogni testata risente della rete delle influenze e dei collaboratori, con un'attenzione sempre maggiore alle immagini. E. STEAD mette in luce a questo proposito "La Revue illustrée" di Ludovic Baschet, il cui figlio René sarà il direttore dell'"Illustration" a partire dal 1904. Si tratta di riviste con un ruolo centrale europeo, che fondono modelli inglesi e tedeschi e si basano su professionisti di alto livello. Il modello si declina in forme diverse nelle riviste delle diverse aree nazionali, mostrando che c'è tutt'altro che un'uniformazione, ma la circolazione delle idee resta ampia, coinvolgendo anche gli Stati Uniti. Le riviste moderniste anglo-americane, di base a Parigi, inventano un'identità culturale americana. Inoltre la rete delle riviste indica una continuità e un'evoluzione della storia letteraria dove Dada e surrealismo non si pongono come rotture ma si inseriscono naturalmente nel corso del dibattito estetico. Il lavoro di Daphné de MARNEFFE, che propone di disporre le riviste secondo un doppio asse diacronico e sincronico, mette bene in risalto l'importanza delle riviste di seconda linea nell'elaborazione delle teorie dadaiste e surrealiste e permette di cogliere l'ampiezza del dibattito. 
Il concetto di rete porta a interrogarsi sulla distinzione tra rete, comunità, influenza, grado di socievolezza. Alcune riviste, come Le Saint-Graal (1892-1899) dominata da Emmanuel Signoret e apparentemente circoscritta a lui, è in realtà diffusissima negli ambienti cattolici, felibristi e idealisti grazie alle riviste "amiche". A volte manca una reciprocità, come per la rivista americana francofila "Yellow Book", che non ha una grande diffusione in Francia; mentre la rivista catalana Pel \& Ploma (1899-1902) ha una platea europea. "Vers et prose" di Paul Fort è punto di convergenza di reti. Per la diffusione di "Simplicissimus" (1896-1944) è molto vantaggioso il fatto che l'editore si rifugi in Francia, rinforzando così le sue relazioni. I gruppi anarchici hanno molto interesse a stabilire contatti europei, e nelle loro riviste l'immagine acquista un ruolo preponderante.

9 Sono molti dunque i legami che stringono insieme le Petites revues: informali o istituzionali, estetici e politici, ambizioni internazionali e convergenze nazionali. La presenza di ritratti, così come le posizioni manifestarie, favoriscono l'identificazione e i rapporti.

10 A cavallo del secolo nascono anche riviste specializzate (sul teatro, l'arte, la letteratura e il cinema) che rimettono in causa i limiti disciplinari, oppure, come per le riviste sovietiche degli anni Venti sulla fotografia, ricercano una legittimazione ideologica oltre che artistica. Le riviste di fotografia e di cinema compiono anche il fondamentale lavoro di integrazione nell'arte di aree che non erano ancora sempre ritenute artistiche.

11 L'ultima sezione del volume è dedicata all'insieme dei tentativi di classificazione contemporanei, che si stanno sviluppando da più parti, a cominciare dalle azioni della BNF, il blog di Mikaël Lugan, i siti di Christian Buat e Vincent Gogibu (www.remydegourmont.org) e di Julien Schuh (http://prelia.fr).

Hanno partecipato alla miscellanea, in ordine di intervento: Diana COOPER-RICHET, JeanPierre BACOT, Eliseo tRENC, Sarah AL-MATARY, Marie-Linda ORTEGA, Laurence DANGUY, Evanghelia STEAD, Laurence DANGUY, Vanja STRUKelJ, Francesca zanella, Daphné DE MARNEFFE, Alessia KALANTZIS, Elisa GRILLI, Vincent gogibu, Blaise wiLFERT-PORTAL, Fabienne FRAVALO (con due articoli) Adriana SOTROPA, Anne REYNES-DELOBEL, Jean-Louis MEUNIER, Michel RAPOPORT, Sarah JAMMES, Claire POPINEAU, Mateusz CHMURSKI, Jean-Claude GARDES, Ursula E.Koch, Xavier galmiche, Audrey ziane, Céline mansanti, Yoan VÉRILHAC, Pierre PINCHON, Dorothée PAUVERT-RAIMBAULt, Julien SCHUH, Markéta THEINHARDT, Laurent BIHL, Marco consolini, Sophie lucet, Romain Piana, Paul edWARds, Ada ACKerman, Christophe GAUTHIER, Jean-Didier WAGNEUR, Mikaël LUGAN, Lorraine JANZEN KOOISTRA. 